\title{
Polarity Switchable Photovoltages in Miscut $\mathrm{La}_{0.67} \mathrm{Ca}_{0.33} \mathrm{MnO}_{3}$ Films
}

\author{
J. Wang, ${ }^{1,2}$ H. Ni, ${ }^{2,3}$ K. Zhao, ${ }^{1,2,3,4}$ Y.-C. Kong, ${ }^{3}$ H. K. Wong, ${ }^{3}$ and S. X. Zhong ${ }^{2}$ \\ ${ }^{1}$ State Key Laboratory of Heavy Oil Processing, China University of Petroleum, Beijing 102249, China \\ ${ }^{2}$ College of Science, China University of Petroleum, Beijing 102249, China \\ ${ }^{3}$ Department of Physics, Chinese University of Hong Kong, Hong Kong \\ ${ }^{4}$ International Center for Materials Physics, Chinese Academy of Sciences, Shenyang 110016, China
}

Correspondence should be addressed to K.Zhao, zhk@cup.edu.cn

Received 26 May 2011; Accepted 19 October 2011

Academic Editor: Vincenzo Augugliaro

Copyright () 2012 J. Wang et al. This is an open access article distributed under the Creative Commons Attribution License, which permits unrestricted use, distribution, and reproduction in any medium, provided the original work is properly cited.

\begin{abstract}
We report the transient laser-induced anomalous photovoltaic effect in the $\mathrm{La}_{0.67} \mathrm{Ca}_{0.33} \mathrm{MnO}_{3}$ film grown on miscut $\mathrm{LaSrAlO}_{4}$ (001) substrate under $248 \mathrm{~nm}$ pulsed laser irradiation at ambient temperature without any applied bias. A photovoltaic pulse signal was observed when the $\mathrm{La}_{0.67} \mathrm{Ca}_{0.33} \mathrm{MnO}_{3}$ film was irradiated directly, and the signal polarity was reversed when the sample was irradiated through the $\mathrm{LaSrAlO}_{4}$ substrate, while the signal recorded between the two electrodes on the surface of $\mathrm{LaSrAlO}_{4}$ was not reversed when the sample was irradiated through $\mathrm{La}_{0.67} \mathrm{Ca}_{0.33} \mathrm{MnO}_{3}$ film rather than at the surface of $\mathrm{LaSrAlO}_{4}$. The switchable signal polarity provides a potential application of miscut manganite films as optical components. A possible mechanism is introduced to explain the experiment results.
\end{abstract}

\section{Introduction}

Up to now, investigations of manganites have been focused on their interesting properties, including ferroelectric, ferromagnetic, giant magnetoresistance, and superconducting and electrooptic properties [1-6]. Several researches about photovoltaic effect and thermoelectric response were carried out for exploring their potential applications in detector [710]. Anomalous photovoltaic effect has been observed in $\mathrm{La}_{0.8} \mathrm{Sr}_{0.2} \mathrm{MnO}_{3}$ films grown on $\mathrm{SrTiO}_{3}(001)$ substrates. The research results provided the anisotropy of the thermoelectric power [11].

It is known that heating the surface of a thin film grown on miscut substrate by the absorption of radiation establishes a temperature gradient $\nabla T$ perpendicular to the film surface. Due to the Seebeck effect, a thermoelectric field, $E=S \nabla T$, is generated with the Seebeck tensor $S$ [12]. The lateral voltage can be written as

$$
U_{l}=\frac{l\left(S_{a b}-S_{c}\right) \sin (2 \theta) \Delta T}{2 d},
$$

where $l$ is the irradiated length by the laser beam between two electrodes, $S_{a b}$ and $S_{c}$ are the Seebeck coefficients of the crystalline $a b$ plane and along the $c$ axis, respectively, $\theta$ is the tilting angle between the $c$ axis and the direction of laser irradiation, $\Delta T$ is the temperature difference between film surface and film bottom, and $d$ is the film thickness.

In this paper, we focus on the polarity-switchable signal which arose in the $\mathrm{La}_{0.67} \mathrm{Ca}_{0.33} \mathrm{MnO}_{3}$ film (LCMO) grown on miscut $\mathrm{LaSrAlO}_{4}$ (LSAO) (001) substrate under $248 \mathrm{~nm}$ pulsed laser irradiation at ambient temperature without any applied bias. We not only observed a photovoltaic signal produced in LCMO film that was reversed when the sample was irradiated through the substrate rather than at LCMO film, but also found a tiny signal generated in LSAO substrate, which was not reversed when the incident light irradiated the substrate in the opposite direction. The possible mechanism introduced to explain the experiment results provides a potential application of manganite films in optical components.

\section{Experimental}

Epitaxial LCMO film with a thickness $d_{1}$ of about $120 \mathrm{~nm}$ was deposited on the LSAO substrate with a thickness $d_{2}$ of $0.5 \mathrm{~mm}$ and an intentional $10^{\circ}$ vicinal cut toward the [010] direction by facing-target sputtering technique. The 

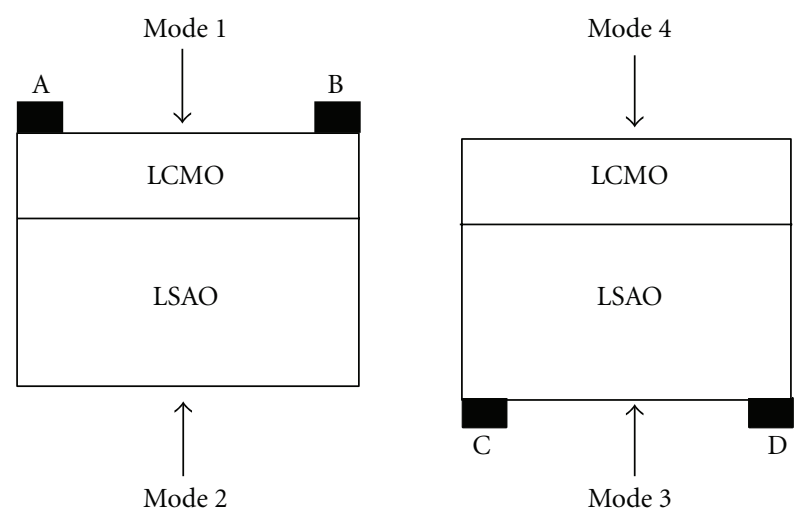

FIGURE 1: Schematic illustration of photoelectrical experiment on LCMO/LSAO sample.

substrate was kept at $680^{\circ} \mathrm{C}$ with the oxygen pressure of 30 mTorr during deposition. After the deposition, the vacuum chamber was immediately back-filled with 1 atm oxygen gas. The LCMO film was then cooled to room temperature with the substrate heater power cut-off. The structure of the sample was characterized by X-ray diffraction (XRD).

Figure 1 shows the schematic illustrations of photovoltaic response measurement. LCMO/LSAO sample of $5 \times 2.5 \mathrm{~mm}^{2}$ geometry was used with two in-plane colloidal silver electrodes separated by $3 \mathrm{~mm}$ being individually placed on the surface of the LCMO film and LSAO substrate as displayed in Figure 1. Modes 1 and 4 (Modes 2 and 3) stand for irradiating the sample through LCMO film (LSAO substrate) with electrodes on LCMO film and LSAO substrate, respectively. The anode was connected with A (and C), and the cathode was connected with B (and D). The electrodes were always kept in the dark to prevent the generation of any electrical contact photovoltaic effect. The $248 \mathrm{~nm} \mathrm{KrF}$ excimer laser (pulse duration of $20 \mathrm{~ns}$, repetition rate of $1 \mathrm{~Hz}$ ) was used as the light source at ambient temperature in air with a pulse energy of $2.88 \mathrm{~mJ}$. The waveform was recorded by a sampling oscilloscope terminated into $1 \mathrm{M} \Omega$.

\section{Results and Discussion}

The XRD $\theta-2 \theta$ scan curve of the LCMO/LSAO is presented in Figure 2, where the [001] axis was aligned carefully. Except for the diffraction peaks of LSAO (00l) and LCMO $(h 0 h)$, there are no diffraction peaks from impurity phases or randomly oriented grains, indicating that the LCMO film is a single phase and [202] oriented.

Figure 3 displays the photovoltaic responses of LCMO/LSAO under the $248 \mathrm{~nm}$ pulsed laser irradiation without any applied bias. Two significant characteristics of the photovoltaic signals were observed: (i) the polarity of the signal gotten from LCMO film switched when the sample was irradiated through the substrate rather than at the film surface (Figure 3(a)). While no reversed signal polarity arose for modes 3 and 4 (Figure 3(b)). (ii) The order of the peak photovoltage (Up) according to intensity is $\mathrm{U}_{1}>\mathrm{U}_{2}>\mathrm{U}_{3}>$

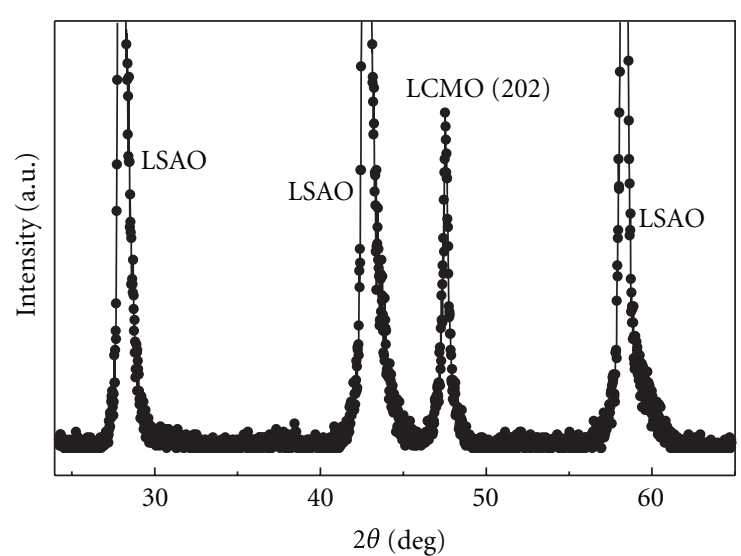

FIGURE 2: XRD pattern of LCMO/LSAO sample.

$\mathrm{U}_{4}$, where $\mathrm{U}_{1}, \mathrm{U}_{2}, \mathrm{U}_{3}$, and $\mathrm{U}_{4}$ correspond to the peak photovoltage for modes $1,2,3$, and 4 . The maximum Up of $0.476 \mathrm{~V}$ was observed with a rising time of $140 \mu \mathrm{s}$ and a full width at half maximum (FWHM) of $29 \mu$ s in mode 1 . While the smallest Up of $0.013 \mathrm{~V}$ was observed in mode 4 , almost 40 times lower than that of mode 1.

To investigate the anomalous photovoltaic effect, we measured the absorption spectra of LCMO/LSAO and LSAO single crystal as presented in Figure 4 . The absorption edge of LSAO crystal is located at $253 \mathrm{~nm}$ in agreement with its band gap of $5.3 \mathrm{eV}$, indicating that nonequilibrium charge carries in LSAO crystal can be created under $248 \mathrm{~nm}$ ultraviolet irradiation. However, the substrate thickness $(0.5 \mathrm{~mm})$ is much longer than the diffusion length of the charge carries in the LSAO side. In addition, due to the strong absorption in LSAO substrate, few photons can inject into the LCMO layer. As a result, a smaller photovoltage presents for mode 2 compared with that for mode 1.

Concerning to illustrate the generation of polarity switchable photovoltages, several researches focused on changing film thickness and regulating the incident angle were carried out [13-16]. These researches involve both tilted films and single crystals where the Seebeck effect is widely used. The reversal signal generated in LCMO film under working 


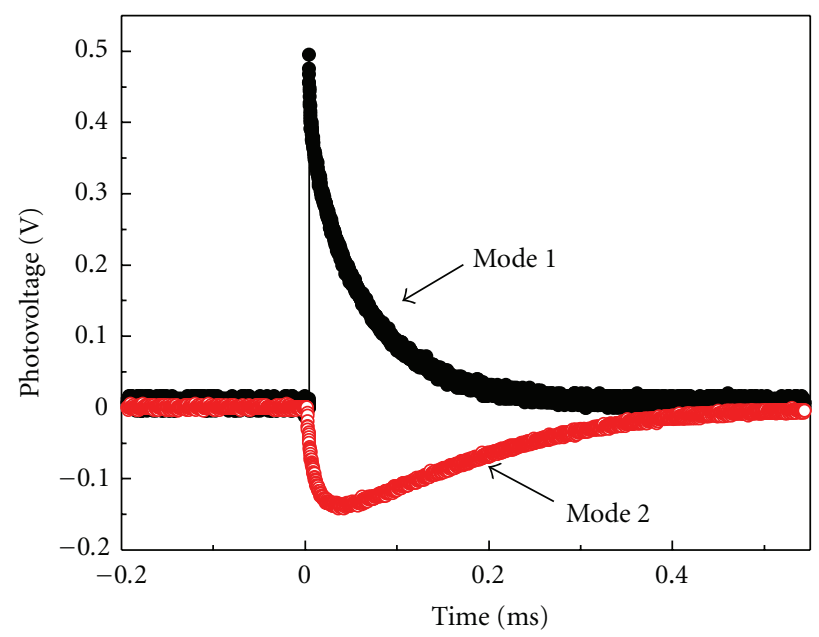

(a)

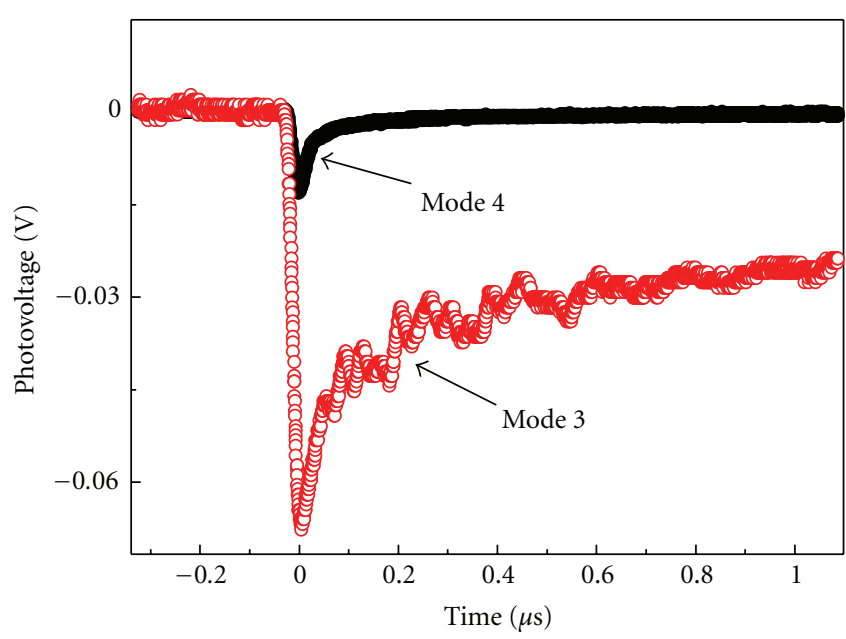

(b)

FIgURE 3: Typical photovoltaic pulses as functions of time for LCMO/LSAO sample.

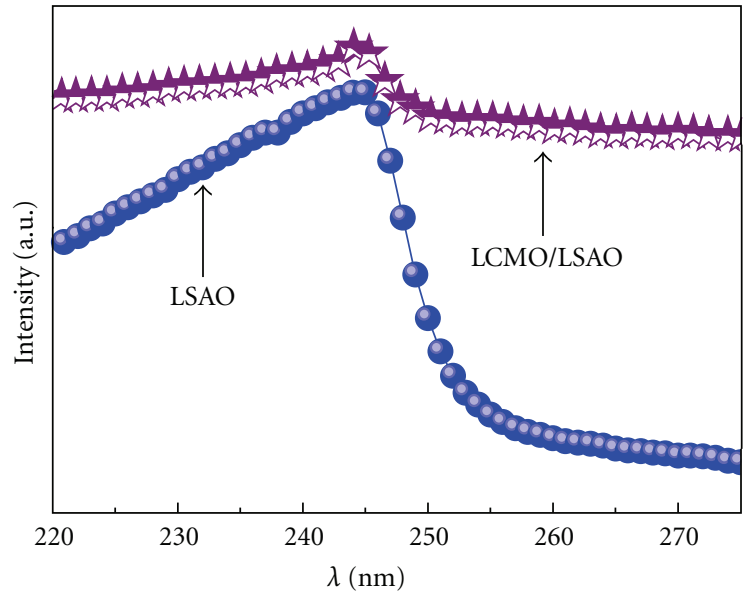

FIGURE 4: The absorption spectra of the LCMO/LSAO sample and LSAO single crystal.

mode 2 is in accordance with our previous study [11], which was attributed mainly to the $\nabla T$ reversal between the front and back end of LCMO film. In order to explain the experiment results shown in Figure 3, the schematic model of the procedure of induced charge and Seebeck processes is built in Figure 5. Due to the Seebeck effect, a positive potential difference $U_{+\mathrm{AB}}^{1}$ along the LCMO film arises when the laser irradiates the sample in the air/LCMO-direction (mode 1). The photo-induced charge carries distribution in LCMO film induces a reversed charge carries distribution along the back-face of LSAO owing to the capacity effect of dielectric, which leads to a negative potential difference $U_{-\mathrm{CD}}^{4}$ (mode 4).

Irradiating the LSAO substrate directly leads to a negative $U_{-\mathrm{CD}}^{3}$ along their surface (mode 3 ), which induced reversed charge distribution on the LCMO/LSAO interface due to the capacity effect and resulted into a positive $U_{+\mathrm{AB}}$ in LSMO film. However, a negative lateral voltage $U_{-\mathrm{AB}}$ along the LCMO is generated due to reversal of the $\nabla T$ in the thickness direction for mode 3 . Thus there exist two opposite photovoltages in LCMO. $U_{+\mathrm{AB}}$ is much smaller than $U_{-\mathrm{AB}}$ because the induced charge on the interface produced by LSAO dielectrics is an extremely small amount compared to that from the reversed $\nabla T$ in LCMO. In sum, a negative $U_{-A B}^{2}$, equal to $U_{-A B}+U_{+A B}$, is generated along the surface of LCMO film (mode 2).

Generally speaking, the polarity of signal generated in LCMO film (modes 1 and 2) is reversed mainly owning to the $\nabla T$ reversal between the front and back end of LCMO film according to Seebeck effect. And two different physical mechanisms, capacity effect of dielectric and Seebeck effect, determine the conformity of photovoltaic polarity under working modes 3 and 4 . In addition, the polarity of photovoltage generated under working mode 3 is reversed, comparing with that under mode 1 , due to the supplementary tilting angle $\theta$ and connection pattern of electrodes.

It is easy to understand that the absolute value of $U_{+\mathrm{AB}}^{1}$ is larger than $U_{-\mathrm{AB}}^{2}$ because $U_{+\mathrm{AB}}$ did much to offset $U_{-\mathrm{AB}}^{2}$. Since the thermoelectric field leading to $U_{-\mathrm{CD}}^{3}$ is much larger than the electric field generated by induced charge of LSAO dielectrics, $U_{-\mathrm{CD}}^{3}$ is larger than $U_{-\mathrm{CD}}^{4}$. The potential 


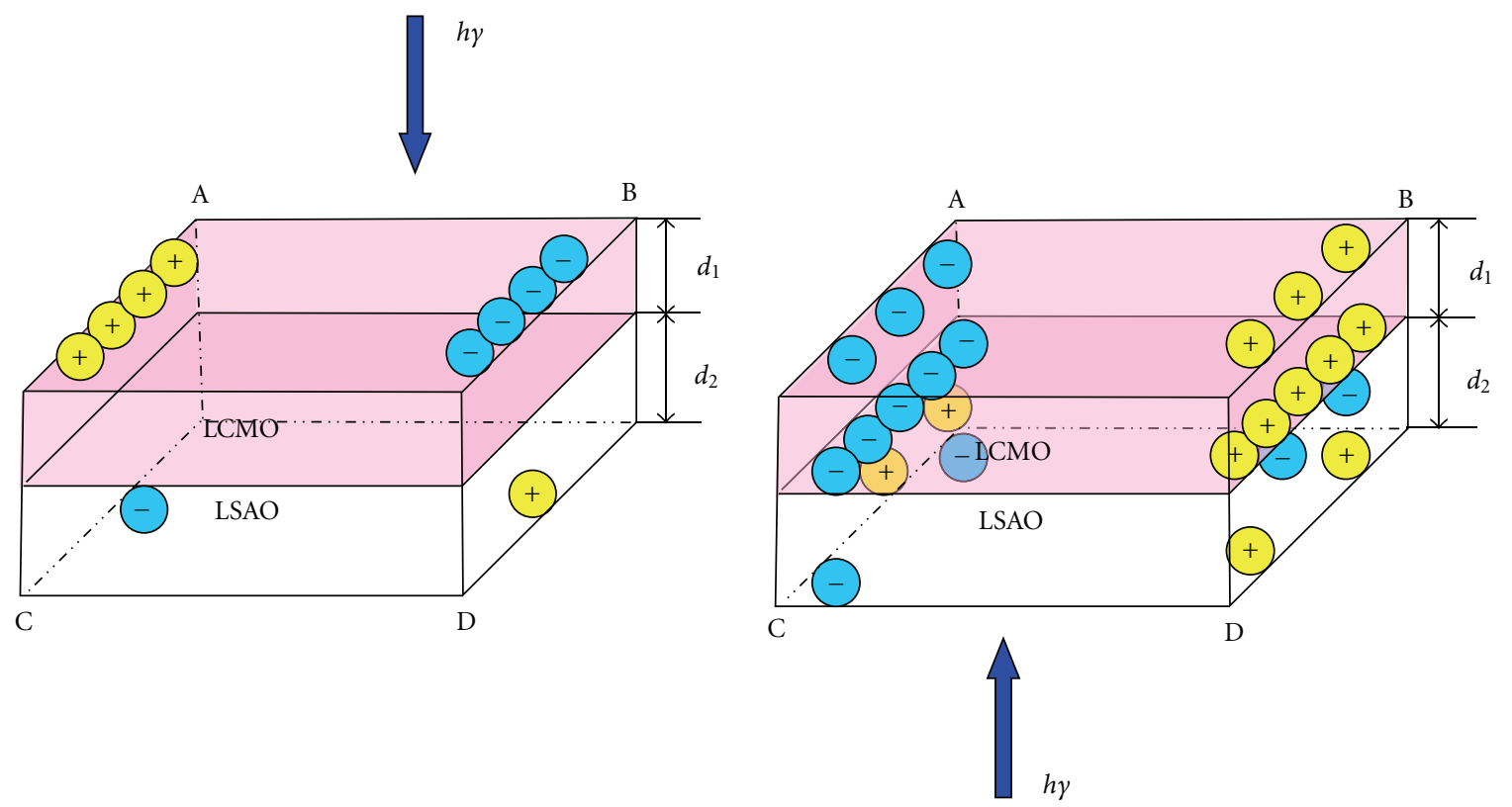

Figure 5: The schematic drawing explaining carries distribution under opposite irradiation directions.

difference produced between A and B on LCMO surface is much larger than that between $\mathrm{C}$ and D on LSAO surface because of the significant difference of thicknesses between LCMO and LSAO $\left(d_{1} \ll d_{2}\right)$ according to (1).

\section{Conclusions}

In summary, we have observed the lateral photovoltaic effects in the LCMO thin film and LSAO substrate under the $248 \mathrm{~nm}$ pulse laser irradiation. The signal polarity inversion shows dependency of irradiation direction and electrode positions. That provides a potential application of the manganite film for photodetector.

\section{Acknowledgments}

This work has been supported by NCET, RFDP, Direct Grant from the Research Grants Council of the Hong Kong Special Administrative Region (Grant no. C001-2060295), and Foresight Fund Program from China University of Petroleum.

\section{References}

[1] T. Choi, S. Lee, Y. J. Choi, V. Kiryukhin, and S. W. Cheong, "Switchable ferroelectric diode and photovoltaic effect in $\mathrm{BiFeO}_{3}$," Science, vol. 324, no. 5923, pp. 63-66, 2009.

[2] J. Badro, J. P. Rueff, G. Vankó, G. Monaco, G. Fiquet, and F. Guyot, "Electronic transitions in perovskite: possible nonconvecting layers in the lower mantle," Science, vol. 305, no. 5682, pp. 383-386, 2004.

[3] C. C. Homes, T. Vogt, S. M. Shapiro, S. Wakimoto, and A. P. Ramirez, "Optical response of high-dielectric-constant perovskite-related oxide," Science, vol. 293, no. 5530, pp. 673676, 2001.
[4] D. D. Fong, G. B. Stephenson, S. K. Streiffer et al., "Ferroelectricity in ultrathin perovskite films," Science, vol. 304, no. 5677, pp. 1650-1653, 2004.

[5] N. V. Volkov, C. G. Lee, P. D. Kim, E. V. Eremin, and G. S. Patrin, "Optically driven conductivity and magnetoresistance in a manganite-based tunnel structure," Journal of Physics D, vol. 42, no. 20, Article ID 205009, 2009.

[6] H. B. Lu, K. J. Jin, Y. H. Huang et al., "Picosecond photoelectric characteristic in $\mathrm{La}_{0.7} \mathrm{Sr}_{0.3} \mathrm{MnO}_{3} \mathrm{Si}$ p-n junctions," Applied Physics Letters, vol. 86, no. 24, Article ID 241915, pp. 1-3, 2005.

[7] X. M. Li, K. Zhao, H. Ni et al., "Voltage tunable photodetecting properties of $\mathrm{La}_{0.4} \mathrm{Ca}_{0.6} \mathrm{MnO}_{3}$ films grown on miscut $\mathrm{LaSrAlO}_{4}$ substrates," Applied Physics Letters, vol. 97, no. 4, Article ID 044104, 2010.

[8] K. W. Liu, J. G. Ma, J. Y. Zhang et al., "Ultraviolet photoconductive detector with high visible rejection and fast photoresponse based on $\mathrm{ZnO}$ thin film," Solid-State Electronics, vol. 51, no. 5, pp. 757-761, 2007.

[9] H. Ni, S. Q. Zhao, and K. Zhao, “Thickness-dependent photoresponse characteristics of miscut $\mathrm{LaSrAlO}_{4}$ single crystals for ultraviolet detection," Applied Optics, vol. 49, no. 14, pp. 2635-2638, 2010.

[10] S. Zeuner, W. Prettl, and H. Lengfellner, "Fast thermoelectric response of normal state $\mathrm{YBa}_{2} \mathrm{Cu}_{3} \mathrm{O}_{7-\delta}$ films," Applied Physics Letters, vol. 66, pp. 1833-1835, 1995.

[11] K. Zhao, H. B. Lu, M. He et al., "Anomalous photovoltaic effect in $\mathrm{La}_{0.8} \mathrm{Sr}_{0.2} \mathrm{MnO}_{3}$ films grown on $\mathrm{SrTiO}_{3}(001)$ substrates by laser molecular beam epitaxy," The European Physical Journal, vol. 35, no. 3, pp. 173-176, 2006.

[12] H. Lengfellner, G. Kremb, A. Schnellbögl, J. Betz, K. F. Renk, and W. Prettl, "Giant voltages upon surface heating in normal $\mathrm{YBa}_{2} \mathrm{Cu}_{3} \mathrm{O}_{7-\delta}$ films suggesting an atomic layer thermopile," Applied Physics Letters, vol. 60, no. 4, pp. 501-503, 1992.

[13] H. S. Kwok and J. P. Zheng, "Anomalous photovoltaic response in $\mathrm{YBa}_{2} \mathrm{Cu}_{3} \mathrm{O}_{7}$," Physical Review B, vol. 46, no. 6, pp. 36923695, 1992. 
[14] K. Takahashi, T. Kanno, A. Sakai, H. Adachi, and Y. Yamada, "Gigantic transverse voltage induced via off-diagonal thermoelectric effect in $\mathrm{Ca}_{x} \mathrm{CoO}_{2}$ thin films," Applied Physics Letters, vol. 97, no. 2, Article ID 021906, 2010.

[15] A. Kyarad and H. Lengfellner, "Angle-dependent photovoltaic effect in Al-Si multilayers," Applied Physics Letters, vol. 87, no. 18, Article ID 182113, pp. 1-3, 2005.

[16] K. Zhao, K. J. Jin, Y. Huang et al., "Ultraviolet fast-response photoelectric effect in tilted orientation $\mathrm{SrTiO}_{3}$ single crystals," Applied Physics Letters, vol. 89, no. 17, Article ID 173507, 2006. 


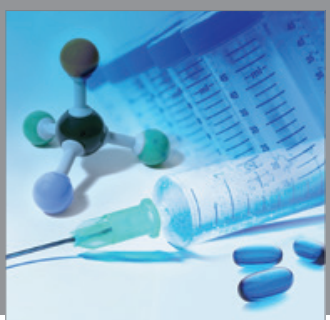

International Journal of

Medicinal Chemistry

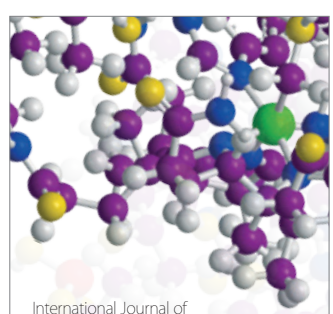

Carbohydrate Chemistry

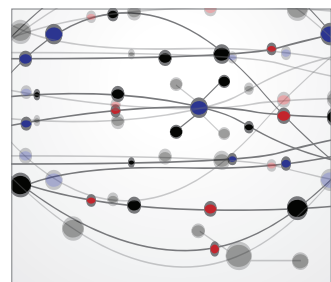

The Scientific World Journal
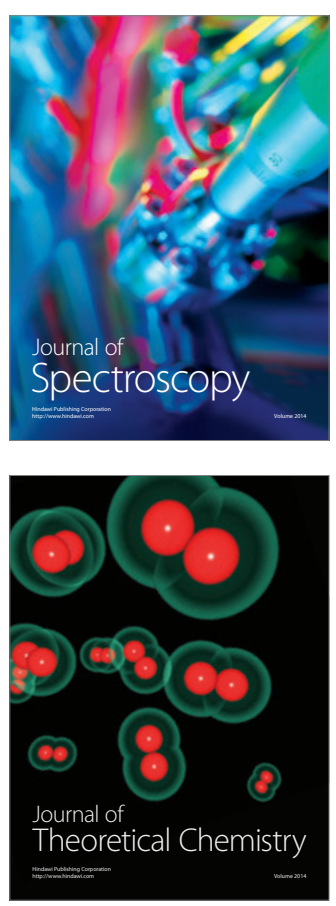
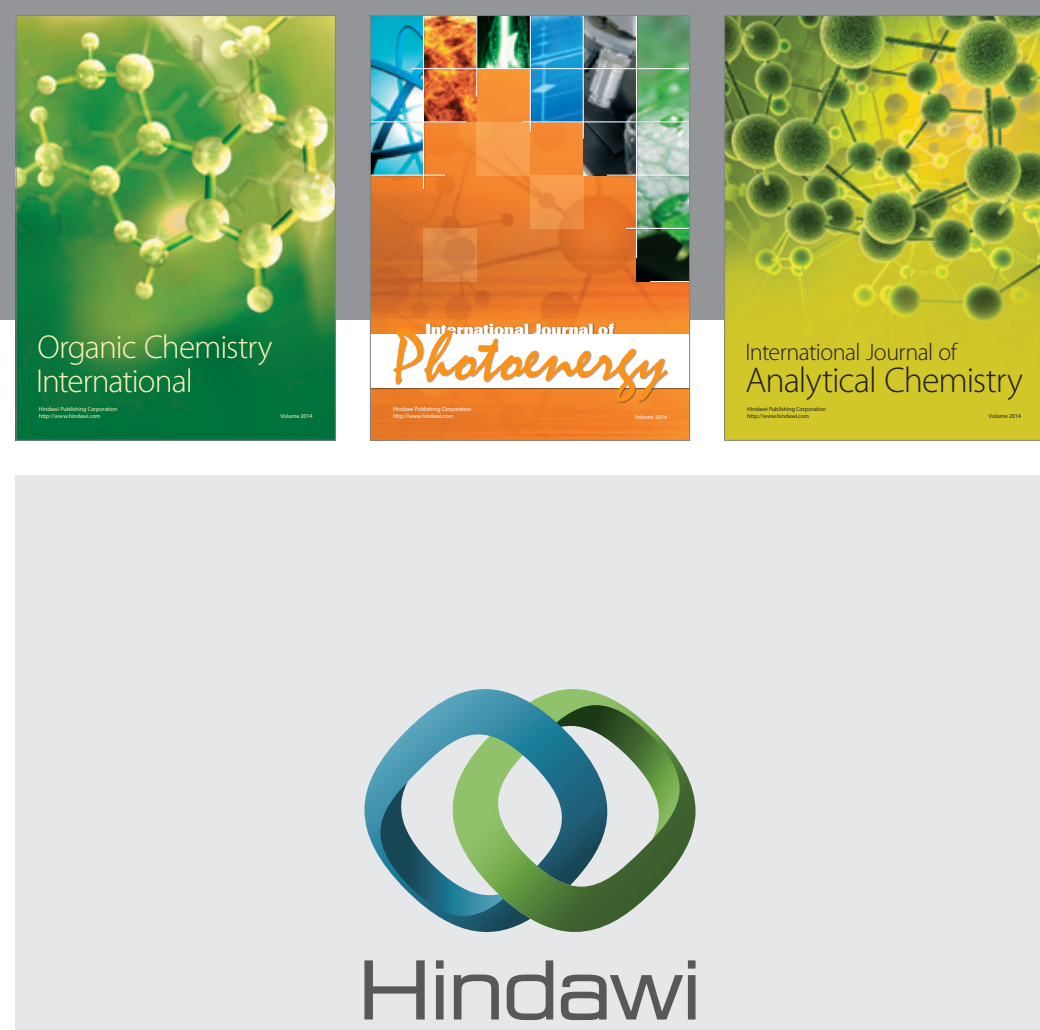

Submit your manuscripts at

http://www.hindawi.com
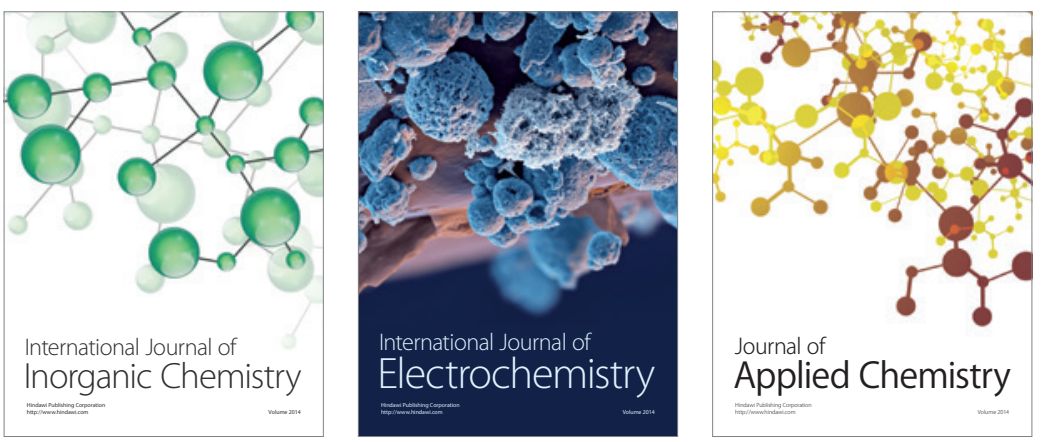

Journal of

Applied Chemistry
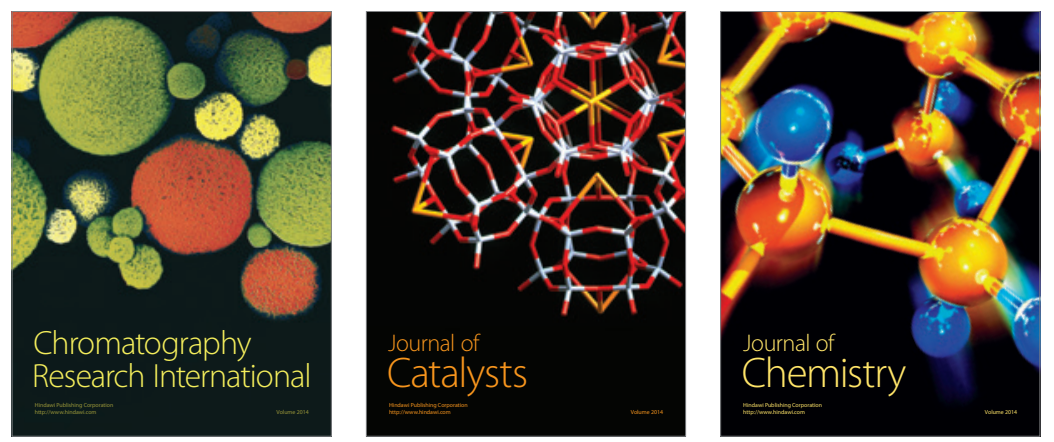
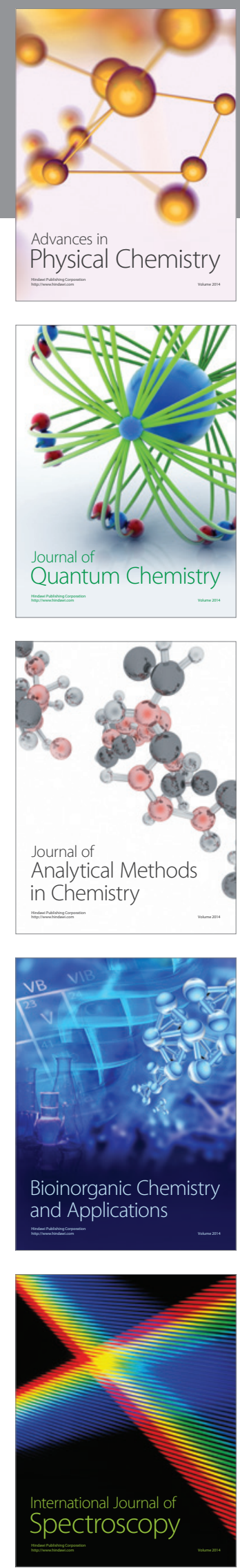